\title{
Pancreatoduodenectomy After Neoadjuvant Chemotherapy for Locally Advanced Pancreatic Cancer in the Presence of an Aberrant Right Hepatic Artery
}

\author{
NICOLAE BACALBASA ${ }^{1,2,3^{*}}$, IRINA BALESCU ${ }^{4}$, MIHAELA VILCU ${ }^{1,2}$, ADINA CROITORU $^{3,5^{*}}$, \\ SIMONA DIMA ${ }^{3}$, VLADISLAV BRASOVEANU ${ }^{3,5}$, IULIAN BREZEAN ${ }^{1,2^{*}}$ and IRINEL POPESCU ${ }^{3,5}$ \\ ${ }^{1}$ Carol Davila University of Medicine and Pharmacy, Bucharest, Romania; \\ ${ }^{2}$ I. Cantacuzino Clinical Hospital, Bucharest, Romania; \\ ${ }^{3}$ Fundeni Clinical Institute - Center of Excellence in Translational Medicine, Bucharest, Romania; \\ ${ }^{4}$ Ponderas Academic Hospital, Bucharest, Romania; \\ ${ }^{5}$ Titu Maiorescu University of Medicine and Pharmacy, Bucharest, Romania
}

\begin{abstract}
Background/Aim: Locally advanced pancreatic head cancer remains an aggressive malignancy with a low likelihood of achieving resectability after neoadjuvant chemotherapy. Resection is even more difficult if anatomical variations of the blood supply are present. Case Report: We present the case of a 62-year-old male diagnosed with locally advanced pancreatic cancer in the presence of an aberrant right hepatic artery originating from the superior mesenteric artery. After completing six cycles of neoadjuvant chemotherapy consisting of irinotecan and oxaliplatin, resectability was achieved, the patient being submitted to pancreatoduodenectomy. Intraoperatively, the presence of an aberrant right hepatic artery originating from the superior mesenteric artery was confirmed. The postoperative course was uneventful, the patient being discharged on the eight postoperative day, while the histopathological studies confirmed the negativity of the resection margins. Conclusion: Resectability can be achieved after neoadjuvant chemotherapy for locally advanced pancreatic cancer. However, attention should be focused on the possibility of the presence of anatomical variations of the pancreatic and liver blood supply.
\end{abstract}

This article is freely accessible online.

*These Authors contributed equally to this study.

Correspondence to: Irina Balescu, Ponderas Academic Hospital, Bucharest, Romania. Tel: +40 724077709, e-mail: irina.balescu@ ponderas-ah.ro

Key Words: Aberrant right hepatic artery, neoadjuvant chemotherapy, resectability.
Pancreatic adenocarcinoma still represents the fourth cause of cancer-related mortality worldwide, and is estimated to become the second cause of cancer-related death by 2030 $(1,2)$. This fact is mainly related to the diagnosis of the disease in advanced stages when massive local invasion or distant metastases are already present. However, cases which are diagnosed in resectable stages might have a chance for long-term survival; therefore, attention was focused on creating neoadjuvant chemotherapy protocols which might increase the resectability rate in such cases (3$5)$. However, in such cases, detailed preoperative imagistic evaluation is mandatory in order to provide an exact description of the anatomical relationship between the tumor and the adjacent vascular structures; furthermore, attention should be focused on determining the possible anatomical variations of the vascular supply of the pancreatic head and of the liver, multiple such situations being described so far (4-8).

We present the case of a 62-year-old patient, initially diagnosed with a locally advanced pancreatic head carcinoma, submitted to neoadjuvant chemotherapy, followed by radical resection. The patient also presented an anatomical particularity which made resection more difficult - the presence of an aberrant right hepatic artery (aRHA) originating from the superior mesenteric artery (SMA).

\section{Case Report}

A 62-year-old male was investigated for jaundice and upper abdominal pain, and was diagnosed at that time by computed tomography with a $5 \mathrm{~cm}$ tumor of the pancreatic head in close contact ( $>180$ degrees) with the superior mesenteric artery and with the portal vein (>180 degrees), which also presented focal narrowing and contour irregularity (Figure 
1). Computed tomography demonstrated the presence of an aRHA originating from the SMA (Figure 2).

The endoscopic ultrasound performed at that time confirmed the tumoral lesion and biopsied it; histopathological studies demonstrated the presence of a well-differentiated tubular pancreatic adenocarcinoma. External biliary drainage was performed, and the patient was addressed to the Oncology Clinic for neoadjuvant chemotherapy. In this respect, six cycles of irinotecan (360 $\mathrm{mg}$ ) and oxaliplatin $(120 \mathrm{mg})$ were administered. At the end of the neoadjuvant chemotherapeutic treatment the patient was resubmitted to a computed tomography which revealed the shrinkage of the tumoral volume (from 5 to $3.5 \mathrm{~cm}$ ), the decrease of the degree of solid soft-tissue contact of the SMA, as well as of the portal vein (Figure 3). Therefore, the patient was submitted to surgery, with the intent of tumoral resection.

Intraoperatively, in order to identify the aberrant arterial structure, we dissected the hepatoduodenal ligament after palpating the presence of a pulsatile structure at this level. Therefore, dissection went further by performing an extended Kocher maneuver which allowed the identification of the SMA and of the aRHA arising at this level. Dissection of the liver pedicle confirmed the absence of a veritable RHA originating from the proper hepatic artery. After dissecting the arterial structures at this level, we dissected the gastroduodenal artery and preserved as much length as possible and confirmed the absence of tumoral involvement at the level of the aRHA. The lymph node dissection was then continued at the level the hilar and aorto-caval regions. After removing the specimen, the reconstruction was created in the traditional manner (Figures 4 and 5). The postoperative evolution was uneventful, the patient being discharged on the eighth postoperative day. The histopathological study confirmed the presence of a moderately differentiated pancreatic adenocarcinoma, all the resection margins being negative.

\section{Discussion}

Normal hepatic arterial supply refers to the presence of a common hepatic artery arising from the celiac trunk, which branches off from the gastroduodenal and the right gastric artery, transforming into the proper hepatic artery, which finally bifurcates into the left and right hepatic arteries (9). However, this situation which is widely recognized as being normal is actually encountered in only $55 \%$ to $75 \%$ of cases $(6,7)$. Among cases presenting anatomical particularities of arterial hepatic vascularization, the most commonly encountered is an aRHA originating from the SMA (6-8). Aberrant hepatic arterial supply has been described in up to $40 \%$ of cases and has been classified according to different authors, the most widely accepted classifications originating from Michels (10) and Hiatt et aI. (11). While Michels' classification was created based on a study of 200 autopsies, Hiatt et al.'s classification was based on 1,000 angiographies. According to both of these authors, the most commonly encountered variation is represented by the presence of aRHA; Michels further classifies this variation into two categories: replaced RHA and accessory RHA. The first refers to cases in which the aRHA originates from the SMA and courses posteriorly, anteriorly or within the pancreatic parenchyma, while the second refers to cases in which the aRHA has a similar course but is found in addition to a normal RHA. According to Michels' study, the former is encountered in $8-14 \%$ of cases, while the latter in up to $7 \%$ of cases. According to Hiatt et al.'s classification, the two subcategories proposed by Michels do not exist; they recognize only a single type of aRHA and describe it in 7$21 \%$ of cases $(9-11)$.

In this respect, anatomical details of these possible variants should be recognized preoperatively whenever hepato-bilio-pancreatic procedures are planned (12-16). As for patients submitted to surgery for pancreatic head cancer, it is well known that these surgical procedures are associated with significant rates of postoperative complications, the most commonly reported being pancreatic or biliary leaks and postoperative hemorrhage, leading to an overall morbidity rate of up to $50 \%$, even in cases with a normal anatomical distribution of the blood supply $(17,18)$.

In consequence, preoperative data regarding the presence of an aRHA play a crucial role in cases in which a pancreatoduodenectomy is planned. In such cases, the presence of an aRHA should be signaled to the surgical team, multiple intraoperative accidents being prevented if these details are known preoperatively. In cases in which an aRHA is present but this fact is not identified preoperatively, there is a higher risk of intraoperative arterial damage; the aRHA might be not recognized properly and might be ligated, predisposing the patient to developing postoperative complications, such as liver or biliary duct ischemia, leading to the development of a further bilio-enteric anastomotic leak, or might be improperly manipulated and damaged, increasing in this way the risk of developing a further pseudo aneurysm which might lead to massive intra-abdominal bleeding. Another issue is represented by the oncological safety of the procedure, the presence of an aRHA leading in certain cases to a less radical surgical procedure, as a result of the attempt to preserve all the encountered aberrant vascular structures (19-21). Moreover, the presence of an aRHA originating from the SMA makes it susceptible to infiltration by tumor of the pancreatic head, making resection much more difficult or even impossible in certain cases (22).

Therefore, in cases in which a pancreatoduodenectomy is planned, the intraoperative finding of this anatomic variation might predispose to further complications during the early postoperative period, as well as an impaired oncological 


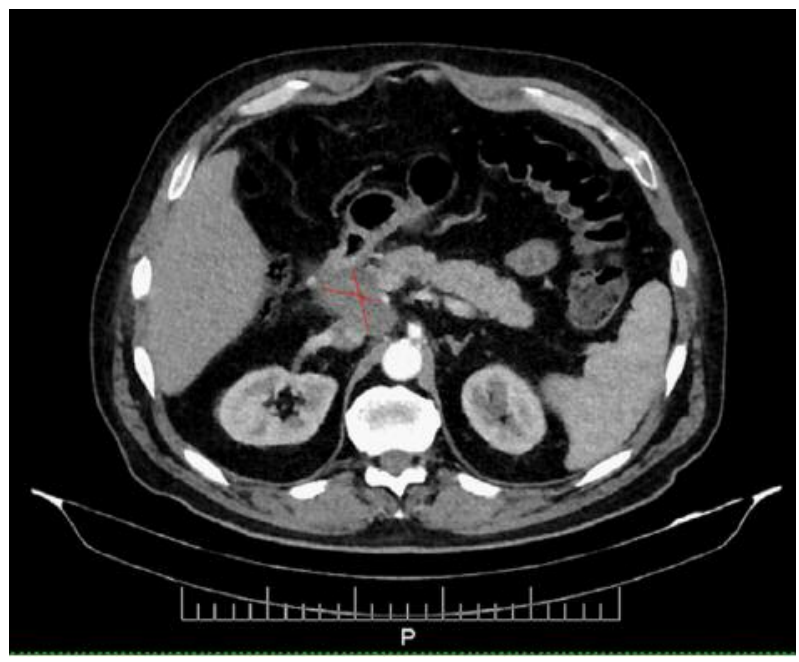

Figure 1. Computed tomography performed before neoadjuvant chemotherapy revealed the presence of a $5 \mathrm{~cm}$ tumor of the pancreatic head in close contact with the superior mesenteric artery and portal vein. The presence of an aberrant right hepatic artery was also described.

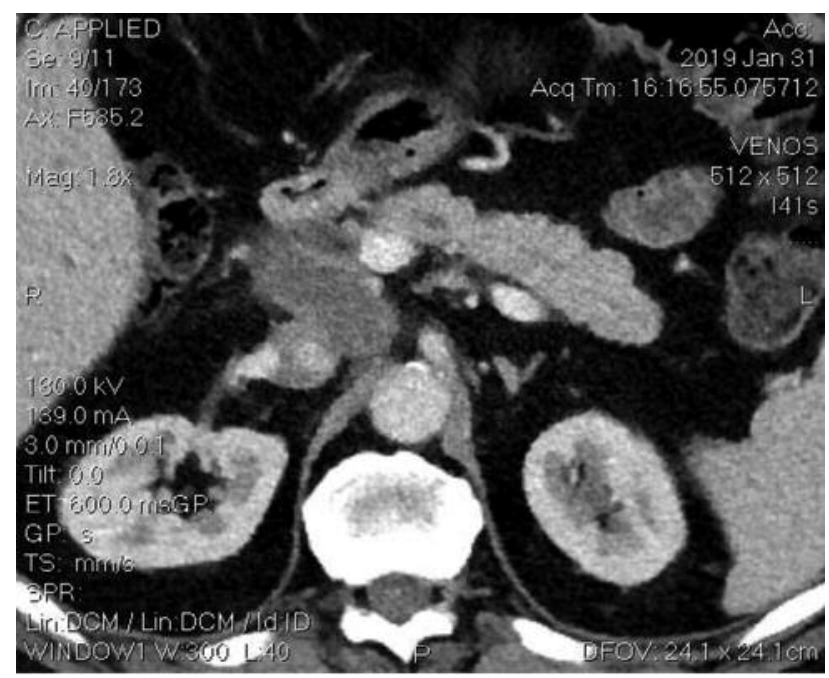

Figure 2. The presence of an aberrant right hepatic artery from the superior mesenteric artery - pre-chemotherapy aspect.

outcome; in consequence, this subject has been widely investigated $(9,22-24)$.

One of the first studies which were conducted on the theme of vascular injury during pancreatoduodenectomy was by Kim et al. and published in 2002 (25). Among the 180 patients submitted to pancreatoduodenectomy for benign, premalignant or malignant diseases, the authors reported a total of 18 cases in which vascular injury took place;

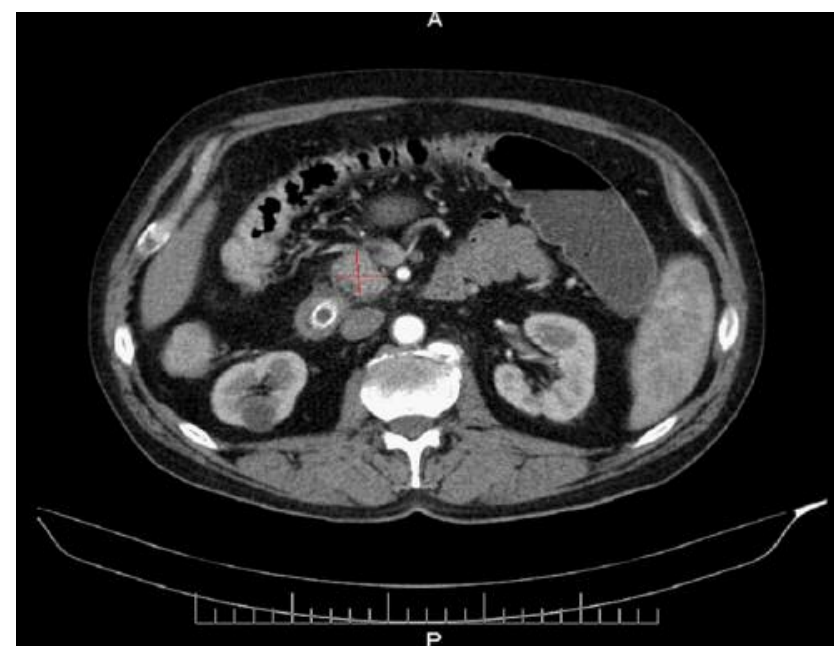

Figure 3. Computed tomography at the end of the neoadjuvant chemotherapy showed tumoral shrinkage to $3.5 \mathrm{~cm}$ and diminished contact between the superior mesenteric artery and the portal vein and the tumor.

however, among these cases, the authors included both patients with venous and arterial injuries. According to their data, an injury of the hepatic artery (left, right, proper or common) was encountered in three cases and consisted of vascular transection in all patients. Regarding the modality of reconstruction, it consisted of saphenous vein interposition graft in two cases, and primary end-to-end anastomosis in the third case. Unfortunately, the exact localization of the injury (left, right, proper or common hepatic artery) is not described. Other encountered injuries involved the SMA, the celiac axis, the superior mesenteric vein and the portal vein. The authors underlined the fact that cases in which vascular injury took place had longer operative times, larger tumors, greater blood loss, longer stay in the Intensive Care Unit and a significantly higher rate of postoperative morbidity and mortality. Poorer long-term survival was also reported among cases in which intraoperative vascular injuries had been occurred (25). However, this study investigated both venous and arterial complications in the same study group; while injuries of the hepatic artery were presented in the same subgroup, no distinction was made in regard to the possible presence of aberrant hepatic arteries.

An interesting study which was conducted on this theme was published in 2011 by Eshuis et al. and included 790 consecutive patients submitted to pancreatoduodenectomy from 1992 to 2009 at the Academic Medical Center in Amsterdam, the Netherlands (9). Among these cases, the authors identified 143 patients presenting an aRHA; in eight of these cases the aRHA was willingly sacrificed for oncological or technical reasons, while in another five cases, 


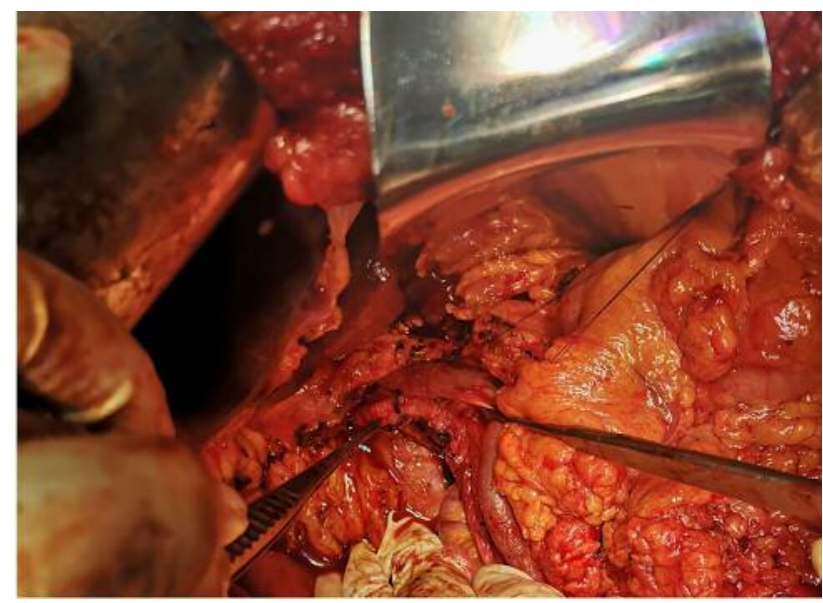

Figure 4. The final aspect after pancreato-duodenectomy. The aberrant right hepatic artery originating from the superior mesenteric artery was entirely preserved.

this artery was accidentally damaged or ligated; in cases in which the aRHA was damaged, it was immediately repaired by suture, while in one case in which it was ligated it was reconstructed by an end-to-end anastomosis. The authors compared the postoperative outcomes of these patients to those reported for the remaining 615 cases which did not present any anatomical variation. They demonstrated that the complications related to the presence of an aRHA such as hepatico-jejunal leak and intraperitoneal bleeding were similar between the two groups; however, among the 13 cases in which the aRHA was corrupted, there were two cases which developed postoperative complications, one of them consisting of delayed fulminant intraperitoneal bleeding, and the other of the development of a large upper abdominal abscess. Short-term mortality was similar between patients presenting normal arterial anatomy and those presenting an aRHA. When it comes to the long-term outcomes, similar rates of survival were observed (the median overall survival being 19.1 months for cases presenting normal anatomy, and 17.9 months for cases diagnosed with aRHA (9). These data demonstrate that the presence of such an anatomical variation should not be considered as a contraindication for pancreatoduodenectomy, similar short-term and long-term outcomes being reported so far.

The issue of aRHA injury during pancreatoduodenectomy was also investigated in a systematic review which was published in 2017 in the Journal of Visceral Surgery (26). The authors included 11 studies, hepatic artery injury during pancreatic surgery being reported in 22 cases; among these patients, anatomical variations of the RHA (presenting an aberrant origin from the SMA) were reported in two cases. In both cases, the injury of the aRHA was diagnosed and repaired intraoperatively and did not lead to liver necrosis.

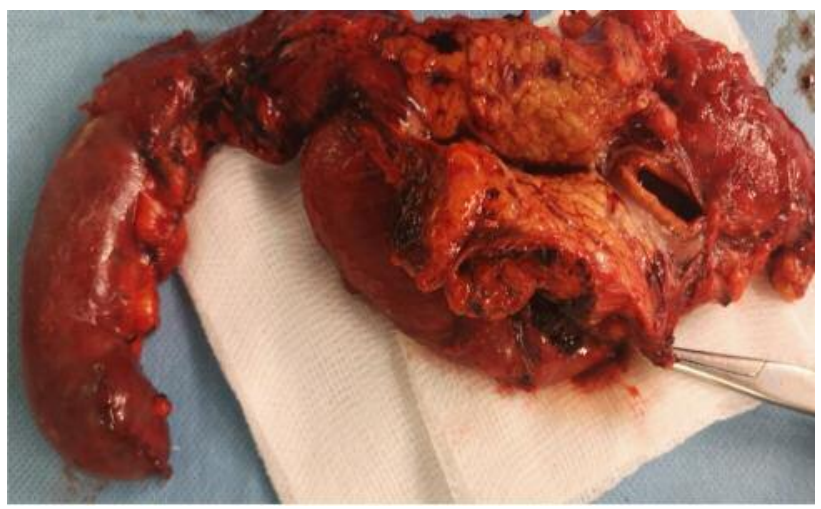

Figure 5. The specimen from pancreatoduodenectomy. Although the patient presented an anatomical variant of arterial distribution, the tumor was completely resected (with negative resection margins).

However, one case which had been submitted to surgery for an ampulloma developed further postoperative complications and necessitated re-operation; finally, the patient died due to fungal sepsis on the 74th postoperative day. The other case was submitted to immediate re-anastomosis and the postoperative outcome was uneventful (26).

The second particularity of our case is represented by the fact that the patient had been initially diagnosed with locally advanced pancreatic cancer, was submitted to neoadjuvant chemotherapy, and finally the tumor became resectable. In the study conducted by Barenboim et al. and published in the European Journal of Surgical Oncology in 2018, the authors investigated the resectability of locally advanced or borderline resectable pancreatic cancer (4). In this analysis, the authors included 30 cases that had been initially diagnosed with locally advanced pancreatic cancer and 23 cases with borderline resectable lesions submitted to neoadjuvant FOLFIRINOX; at the end of the neoadjuvant chemotherapy, only three patients from the group of patients initially diagnosed with locally advanced pancreatic cancer benefited from surgery with curative intent; on the contrary, 20 out of the 23 cases initially diagnosed with borderline resectable lesions were also submitted to surgery. Therefore, the authors concluded that in cases presenting initially with locally advanced pancreatic cancer, resectability can be rarely achieved after neoadjuvant treatment (4). In a similar study conducted in Heidelberg, Germany, a conversion rate to resectability of $29 \%$ after neoadjuvant chemotherapy consisting of FOLFIRINOX protocol was reported for cases initially diagnosed with locally advanced pancreatic cancer; the authors reported no significant postoperative complications in cases in which resectability was achieved after completing the neoadjuvant protocol, demonstrating the 
safety and feasibility of the method. Moreover, the authors underlined that a significant benefit in terms of survival is to be expected if resection is feasible, reporting a mean tumorspecific survival of 31 months for cases submitted to resection, and of only 12 months for those in which resection was not possible (5).

\section{Conclusion}

In rare cases, neoadjuvant chemotherapy might provide a chance for cure in patients with locally advanced pancreatic cancer by tumor shrinkage and achievement of resectability. However, preoperative imaging studies play a central role in such cases, in order to determine the presence of anatomical variants of arterial distribution. Such information is mandatory in order to prevent severe intraoperative and postoperative complications. Although initially considered as representing a contraindication for pancreatoduodenectomy, more recent studies have demonstrated the feasibility of the procedure in the presence of an aRHA and similar oncological outcomes when compared to cases presenting a normal anatomical distribution.

\section{Conflicts of Interest}

The Authors declare no conflicts of interest exist in regard to this study

\section{Authors' Contributions}

NB, SD: Part of the surgical team, prepared the figures. NB: Revised the final draft of the article. IB, MV, VB, IB: Prepared the article and performed the literature review. AC: Oncology consultant. IP: Performed the surgical procedure.

\section{Acknowledgements}

This work was supported by the project entitled "Multidisciplinary Consortium for Supporting the Research Skills in Diagnosing, Treating and Identifying Predictive Factors of Malignant Gynecologic Disorders", project number PN-III-P1-1.2-PCCDI20170833 .

\section{References}

1 Siegel R, Ma J, Zou Z and Jemal A: Cancer statistics, 2014. CA Cancer J Clin 64: 9-29, 2014. PMID: 24399786. DOI: 10.3322/ caac. 21208

2 Rahib L, Smith BD, Aizenberg R, Rosenzweig AB, Fleshman $\mathrm{JM}$ and Matrisian LM: Projecting cancer incidence and deaths to 2030: the unexpected burden of thyroid, liver, and pancreas cancers in the United States. Cancer Res 74: 2913-2921, 2014. PMID: 24840647. DOI: 10.1158/0008-5472.CAN-14-0155

3 Vincent A, Herman J, Schulick R, Hruban RH and Goggins M: Pancreatic cancer. Lancet 378: 607-620, 2011. PMID: 21620466. DOI: $10.1016 / \mathrm{S} 0140-6736(10) 62307-0$
4 Barenboim A, Lahat G, Geva R, Nachmany I, Nakache R, Goykhman Y, Brazowski E, Rosen G, Isakov O, Wolf I, Klausner JM and Lubezky N: Neoadjuvant FOLFIRINOX for locally advanced and borderline resectable pancreatic cancer: An intention to treat analysis. Eur J Surg Oncol 44: 1619-1623, 2018. PMID: 30146251. DOI: 10.1016/j.ejso.2018.07.057

5 Nitsche U, Wenzel P, Siveke JT, Braren R, Holzapfel K, Schlitter AM, Stoss C, Kong B, Esposito I, Erkan M, Michalski CW, Friess $\mathrm{H}$ and Kleeff J: Resectability after first-line FOLFIRINOX in initially unresectable locally advanced pancreatic cancer: A single-center experience. Ann Surg Oncol 22(Suppl 3): S1212S1220, 2015. PMID: 26350368. DOI: 10.1245/s10434-0154851-2

6 Yamamoto S, Kubota K, Rokkaku K, Nemoto T and Sakuma A: Disposal of replaced common hepatic Artery coursing within the pancreas during pancreatoduodenectomy: report of a case. Surg Today 35: 984-987, 2005. PMID: 16249858. DOI: 10.1007/s00595005-3040-5

7 Marin-Gomez LM, Gomez-Bravo MA, Bernal-Bellido C, Alamo-Martinez JM, Suarez-Artacho G and Serrano-DiezCanedo J: Variability of the extrahepatic arterial anatomy in 500 hepatic grafts. Transplant Proc 42: 3159-3161, 2010. PMID: 20970635. DOI: 10.1016/j.transproceed.2010.05.078

8 Cloyd JM, Chandra V, Louie JD, Rao S and Visser BC: Preoperative embolization of replaced right hepatic artery prior to pancreaticoduodenectomy. J Surg Oncol 106: 509-512, 2012. PMID: 22374866. DOI: 10.1002/jso.23082

9 Eshuis WJ, Olde Loohuis KM, Busch OR, van Gulik TM and Gouma DJ: Influence of aberrant right hepatic artery on perioperative course and long-term survival after pancreatoduodenectomy. HPB 13: 161-167, 2011. PMID: 21309932. DOI: 10.1111/j.1477-2574.2010.00258.x

10 Michels NA: Newer anatomy of the liver and its variant blood supply and collateral circulation. Am J Surg 112: 337-347, 1966. PMID: 5917302. DOI: 10.1016/0002-9610(66)90201-7

11 Hiatt JR, Gabbay J and Busuttil RW: Surgical anatomy of the hepatic arteries in 1000 cases. Ann Surg 220: 50-52, 1994. PMID: 8024358. DOI: 10.1097/00000658-199407000-00008

12 Brezean I, Aldoescu S, Catrina E, Fetche N, Marin I and Pacescu E: Gallstone ileus: analysis of eight cases and review of the literature. Chirurgia 105: 355-359, 2010. PMID: 20726301.

13 Bacalbasa N, Brezean I, Anghel C, Barbu I, Pautov M, Balescu $\mathrm{I}$ and Brasoveanu $\mathrm{V}$ : Management of a fulminant upper gastrointestinal bleeding exteriorized through hemobilia due to arteriobiliary fistula between the common bile duct and a right hepatic artery aneurysm - a case report. In Vivo 31: 983-989, 2017. PMID: 28882970.

14 Bacalbasa N, Brezean I, Anghel C, Barbu I, Pautov M, Balescu I and Brasoveanu V: Successful resection and vascular ligation of a large hepatic artery aneurysm - a case report and literature review. In Vivo 31: 979-982, 2017. PMID: 28882969.

15 Bacalbasa N, Balescu I, Tanase A, Pautov M, Brezean I, Vilcu $\mathrm{M}$ and Brasoveanu V: Spleno-pancreatectomy en bloc with parcelar gastrectomy for splenic artery aneurysm - a case report and literature review. In Vivo 32: 915-919, 2018. PMID: 29936480. DOI: 10.21873/invivo.11329

16 Bacalbasa N, Balescu I, Tanase A, Brezean I, Vilcu M and Brasoveanu V: Successful resection of a non-functional paraganglioma with celiac trunk invasion followed by common hepatic artery reimplantation - a case report and literature 
review. In Vivo 32: 911-914, 2018. PMID: 29936479. DOI 10.21873/invivo. 11328

17 de Castro SM, Busch OR, van Gulik TM, Obertop H and Gouma DJ: Incidence and management of pancreatic leakage after pancreatoduodenectomy. Br J Surg 92: 1117-1123, 2005. PMID: 15931656. DOI: $10.1002 /$ bjs.5047

18 de Castro SM, Kuhlmann KF, Busch OR, van Delden OM, Lameris JS, van Gulik TM, Obertop H and Gouma DJ: Delayed massive hemorrhage after pancreatic and biliary surgery: Embolization or surgery? Ann Surg 241: 85-91, 2005. PMID: 15621995. DOI: 10.1097/01.sla.0000150169.22834.13

19 Volpe CM, Peterson S, Hoover EL and Doerr RJ: Justification for visceral angiography prior to pancreaticoduodenectomy. Am Surg 64: 758-761, 1998. PMID: 9697907.

20 Traverso LW and Freeny PC: Pancreaticoduodenectomy. The importance of preserving hepatic blood flow to prevent biliary fistula. Am Surg 55: 421-426, 1989. PMID: 2742226.

21 Shukla PJ, Barreto SG, Kulkarni A, Nagarajan G and Fingerhut A: Vascular anomalies encountered during pancreatoduodenectomy: Do they influence outcomes? Ann Surg Oncol 17: 186-193, 2010. PMID: 19838756. DOI: 10.1245/s10434-009-0757-1

22 Jah A, Jamieson N, Huguet E and Praseedom R: The implications of the presence of an aberrant right hepatic artery in patients undergoing a pancreaticoduodenectomy. Surg Today 39: 669-674, 2009. PMID: 19639433. DOI: 10.1007/s00595-009-3947-3
23 Lee JM, Lee YJ, Kim CW, Moon KM and Kim MW: Clinical implications of an aberrant right hepatic artery in patients undergoing pancreaticoduodenectomy. World J Surg 33: 17271732, 2009. PMID: 19459000. DOI: 10.1007/s00268-009-0063-x

24 Makisalo H, Chaib E, Krokos N and Calne R: Hepatic arterial variations and liver-related diseases of 100 consecutive donors. Transpl Int 6: 325-329, 1993. PMID: 8297461. DOI: 10.1007/ bf00335969

25 Kim AW, McCarthy WJ, III, Maxhimer JB, Quiros RM, Hollinger EF, Doolas A, Millikan KW, Deziel DJ, Godellas CV and Prinz RA: Vascular complications associated with pancreaticoduodenectomy adversely affect clinical outcome. Surgery 132: 738-744, 2002. PMID: 12407360 . DOI: $10.1067 / \mathrm{msy} .2002 .127688$

26 Landen S, Ursaru D, Delugeau V and Landen C: How to deal with hepatic artery injury during pancreaticoduodenectomy. A systematic review. J Visc Surg 154: 261-268, 2017. PMID: 28668523. DOI: $10.1016 /$ j.jviscsurg. 2017.05 .013

Received September 29, 2019

Revised October 16, 2019

Accepted October 18, 2019 